\title{
Neuropsin in mental health
}

\author{
Lina Bukowski ${ }^{1,2}$, Ana M. F. Chernomorchenko ${ }^{1,2}$, Anna Starnawska ${ }^{1,2,3}$, Ole Mors ${ }^{1,4}$, Nicklas H. Staunstrup ${ }^{1,2,3,5^{*}}$ (D, \\ Anders D. Børglum ${ }^{1,2,3}$ and Per Qvist ${ }^{1,2,3}$
}

\begin{abstract}
Neuropsin is a brain-expressed extracellular matrix serine protease that governs synaptic plasticity through activityinduced proteolytic cleavage of synaptic proteins. Its substrates comprise several molecules central to structural synaptic plasticity, and studies in rodents have documented its role in cognition and the behavioral and neurobiological response to stress. Intriguingly, differential usage of KLK8 (neuropsin gene) splice forms in the fetal and adult brain has only been reported in humans, suggesting that neuropsin may serve a specialized role in human neurodevelopment. Through systematic interrogation of large-scale genetic data, we review KLK8 regulation in the context of mental health and provide a summary of clinical and preclinical evidence supporting a role for neuropsin in the pathogenesis of mental illness.
\end{abstract}

Keywords: Neuropsin, KLK8, Mental disorders, Mental health, Biomarker, Human, Depression, Extracellular matrix, LTP, Gene expression

\section{Introduction}

The extracellular matrix (ECM) serine protease neuropsin (also known as NP, PRSS19, BSP1 or TADG14), was named after its apparent neuronal expression and its sequence homology to trypsin [1]. Neuropsin possesses the complete triplet (His-Asp-Ser) of the serine protease domain and exhibits proteolytic activity with a trypsin-like substrate specificity [2]. Identified neuropsin substrates comprise: vitronectin [3]; fibronectin [2]; the cell adhesion molecule L1 (L1CAM) [4]; neuregulin-1 (NRG1) [3]; and the Eph receptor B2 (EphB2) [5], all of which localize to the synapse [6]. Accordingly, and like reported for other trypsin-like serine proteases [7, 8], accumulating evidence support a central role for neuropsin in peri-synaptic proteolysis and structural synaptic plasticity [9]. As several of its proteolytic targets have been linked to neurodevelopmental and mental disorders $[10,11]$, it is thus conceivable that neuropsin governs psychiatry-related synaptic signaling. Here, we review

*Correspondence: nhs@clin.au.dk

2 Department of Biomedicine, Aarhus University, Høegh-Guldbergs Gade 10, Aarhus, Denmark

Full list of author information is available at the end of the article data on regulation of the neuropsin gene $K L K 8$ in the context of mental health and provide a summary of clinical and preclinical evidence supporting a role for neurop$\sin$ in the pathogenesis of mental illness.

\section{Klk8 regulation in the brain}

Human $K L K 8$ mRNA is detected in numerous brain tissues under non-pathological conditions [12] (Fig. 1). Its expression is abundant in the cerebellum throughout life, whereas it peaks postnatally in cortical and limbic tissues, namely in childhood and adulthood [12] (Fig. 1). Age-dependent regional variation in $K l k 8$ expression and translation has additionally been reported in mice. $K l k 8$ mRNA as well as protein levels were shown to decrease in the cerebral cortex with age, while peaking in adults in the olfactory bulb and the hippocampus [13]. Klk8 is susceptible to transcriptional regulation by steroids, as demonstrated by corticosterone in primary cultured hippocampal neurons and in vivo $[14,15]$, and by estradiol in neuronal and microglial cells [16]. Potentially related to this regulation, a sex bias in hippocampal KLK8 expression has been reported at the protein level, with higher expression in healthy adult woman than in men [16]. This, however, is not mirrored at the mRNA level 


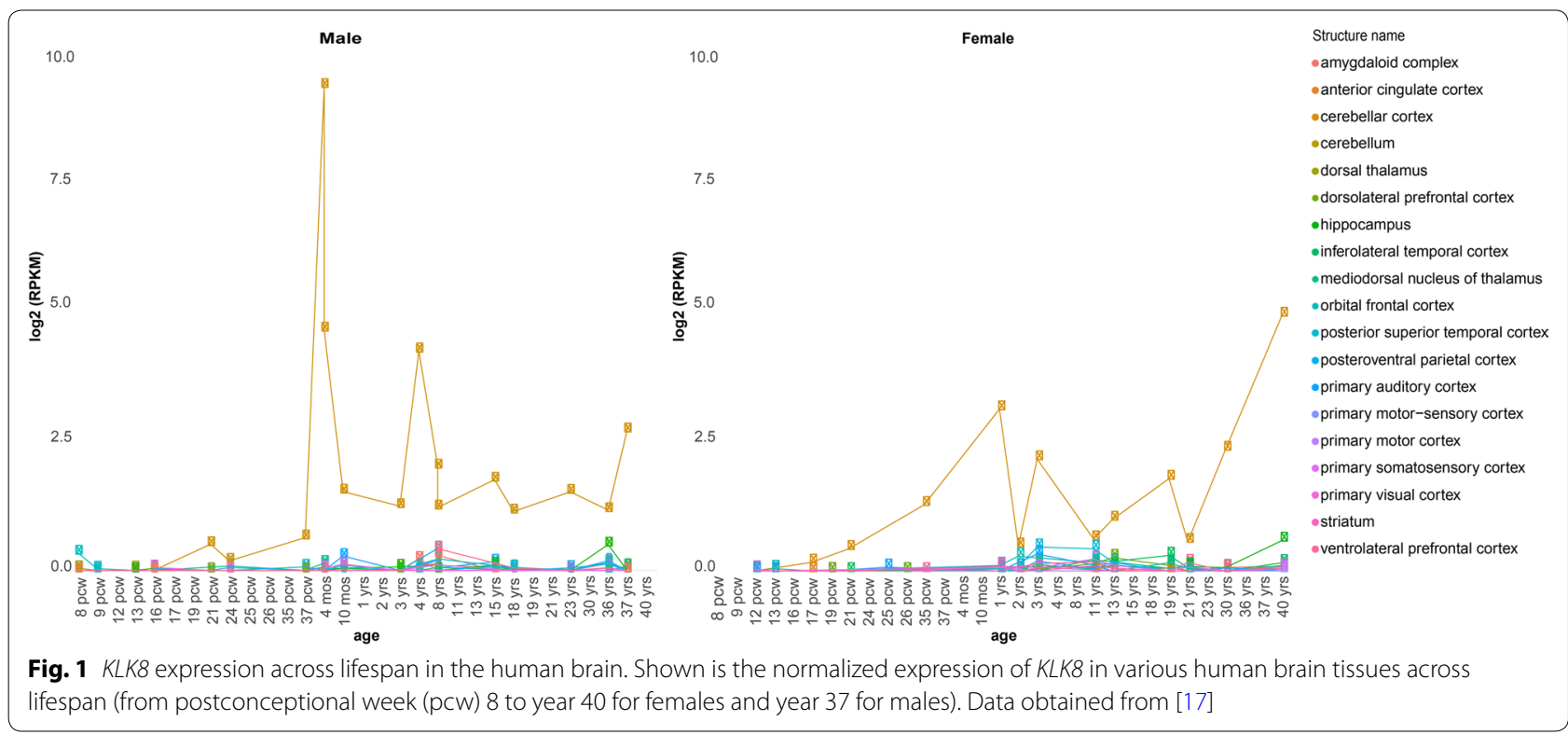

in the Brainspan atlas of the developing human brain [17] (Fig. 1) or in adult hippocampal tissue from healthy donors [18].

The $K L K 8$ transcription start site (TSS) is species and tissue specific $[19,20]$. In the developing mouse cortex and midbrain, the primary sources of $K l k 8$ mRNA are endothelial cells and microglia [21, 22], whereas its expression has been reported in pyramidal neurons of the hippocampal CA1-3 subfields and magnocellular neurons of the lateral/basolateral amygdaloid nucleus on the basis of in situ hybridization immunohistochemistry [1]. Its expression is regulated by neuronal activity $[1,23]$ and also induced in oligodendrocytes of the spinal cord following injury [24]. A re-analysis of data from the most comprehensive human brain single-cell studies, representing several brain tissues and developmental stages, indicate that $K L K 8$ is predominantly expressed in neuronal cells, but generally only in a small fraction of cells (Additional file 1: Table S1). Notably, KLK8 expression was seen in cells derived from temporal cortical tissue in several studies [22, 25], whereas it was not detected in hippocampus-derived cells [18].

While orthologs of $K L K 8$ are found in many species, $K L K 8$ isoforms (type 2-6, Fig. 2a), expressed due to alternative splicing, have only been reported in humans [26-29]. Among these, both type 1 and 2 are abundantly expressed in the central nervous system (CNS) [26].

Although type 1 and type $2 K L K 8$ share the same TSS and lack a splice-form-specific promoter, location of expression appear to be isoform specific. Whereas type 1 is predominantly expressed in the fetal human brain [26], type 2 is detected in embryonic stem cells and embryo brain samples [20], but preferentially expressed in adult brain and particularly in the amygdala and the CA1-3 regions of the hippocampus [26]. Thus, expression of type 2 neuropsin seems to be developmental-stage related. Type $2 K L K 8$ results from an in frame splice site mutation in exon 3 that was fixed during human evolution $[19,30]$. Specifically, a single T-to-A substitution at position 79 in the coding region (Fig. 2a) leads to a novel GAA-containing motif in exon 3 of type $2 K L K 8$, which functions as a splicing enhancer and creates a novel splicing site 8 bp upstream of the locus [19]. Type $2 K L K 8$ is thus identical with type 1 except for a 135 bp ( 45 amino acid) insert located in exon 3 (Fig. 2a) [26]. Interestingly, human type 1 and type $2 K L K 8$ mRNA transcripts produce the same active protease, as the type 2-specific 45-amino acid insertion is located outside the peptide sequence of the mature functional neuropsin protein [20].

$K L K 8$ translation results in a preproneuropsin containing a serine protease peptide, an activity-masking peptide, and a signal peptide [31] (Fig. 3a).

The signal peptide destines the preproneuropsin for translocation to the endoplasmic reticulum and eventually for secretion as a proneuropsin following its removal. Thus, a possible effect of the type 2-specific 45-amino acid insert might involve post-translational modifications (PTMs) or secretory properties. Applying the PTM prediction tool ModPred [32], the insert contained several likely modification sites (Additional file 1: Figure S1). Notably, the insert also resulted in an altered prediction profile of the downstream sequence mutual with type 1 neuropsin (Additional file 2: Table S2). It is thus 


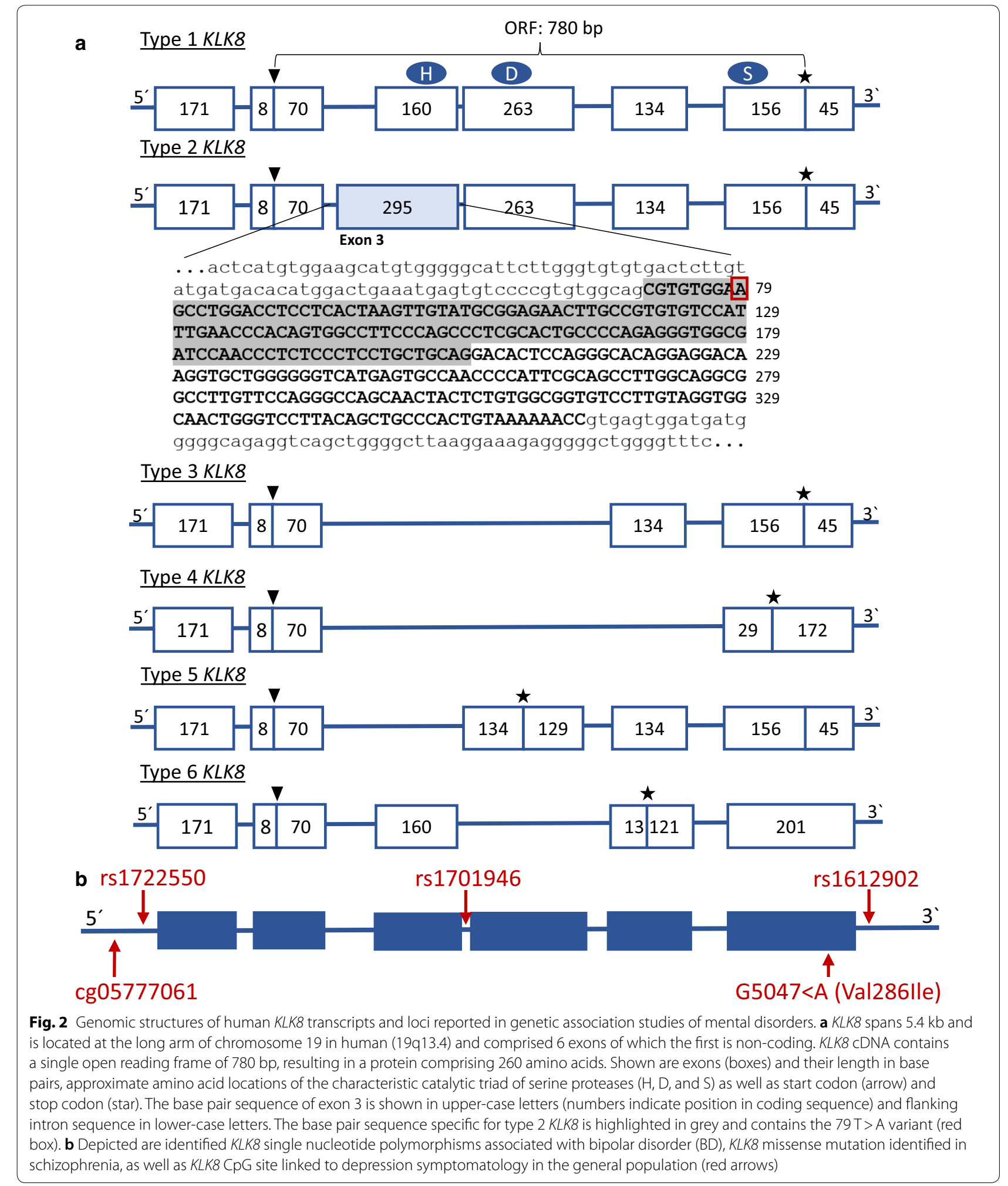

conceivable, that protein folding or protein-protein interactions differ between type 1 and type $2[20,26]$. On the other hand, supporting the notion of different secretory properties, cell-type specific secretion of neuropsin type 1 and 2 has been reported [20]. Proneurop$\sin$ is secreted via the regulated pathway in Neuro2a as 


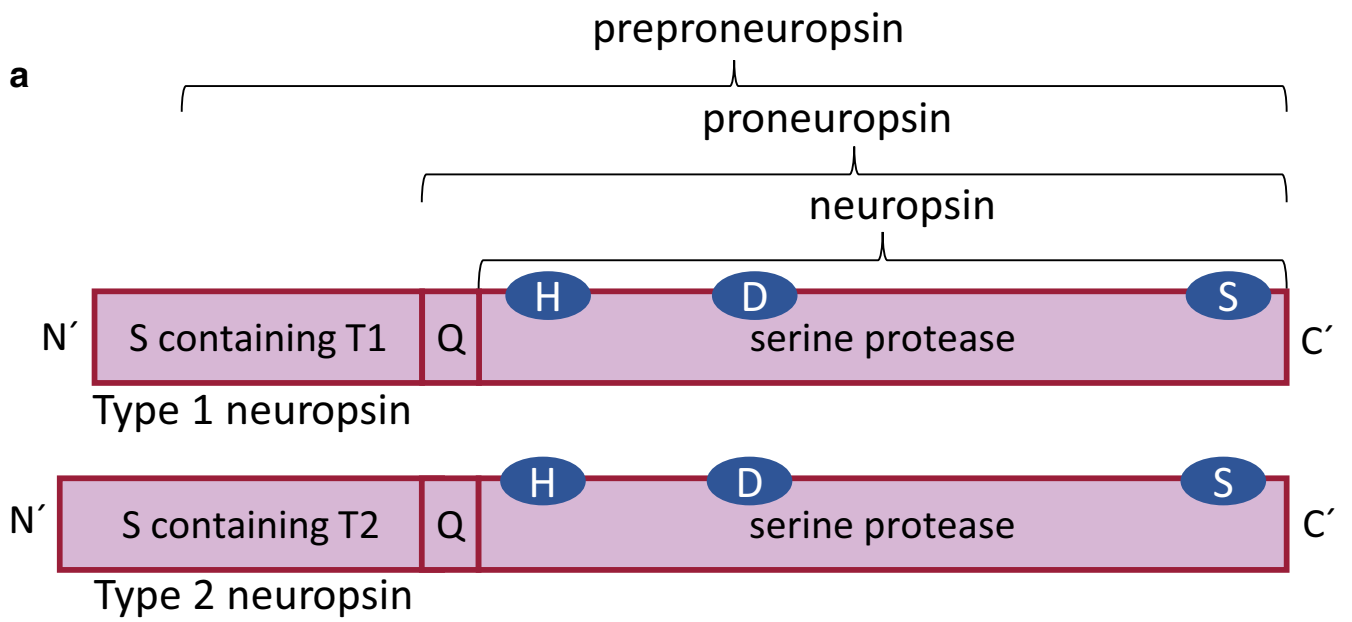

b Type 1 neuropsin ( 260 amino acids)

MGRPRPRAAKTWMFLLLLGGAWAGHSRAQEDKVLGGHECQPHSQPWQAALFQGQQLLCGG VLVGGNWVLTAAHCKKPKYTVRLGDHSLQNKDGPEQE I PVVQS I PHPCYNSSDVEDHNHD LMLLQLRDQASLGSKVKPISLADHCTQPGQKCTVSGWGTVTSPRENFPDTLNCAEVKIFP QKKCEDAYPGQITDGMVCAGSSKGADTCQGDSGGPLVCDGALQGITSWGSDPCGRSDKPG VYTNICRYLDWIKKIIGSKG

\section{Type 2 neuropsin (305 amino acids) MGRPRPRAAKTWMFLLLLGGAWAACGSLDLLTKLYAENLPCVHLNPQWPSQPSHCPRGWR SNPLPPAAGHSRAQEDKVLGGHECQPHSQPWQAALFQGQQLLCGGVLVGGNWVLTAAHCK KPKYTVRLGDHSLQNKDGPEQEIPVVQS IPHPCYNSSDVEDHNHDLMLLQLRDQASLGSK VKPISLADHCTQPGQKCTVSGWGTVTSPRENFPDTLNCAEVKIFPQKKCEDAYPGQITDG MVCAGSSKGADTCQGDSGGPLVCDGALQGITSWGSDPCGRSDKPGVYTNICRYLDWIKKI IGSKG}

Fig. 3 Human neuropsin preproprotein structure. a Shown are: the signal sequence (S) (containing either the type 1-specific amino acid sequence (T1) or type 2-specific amino acid sequence (T2)); the activity-masking peptide (Q) (QGSK in mice and QEDK in human); and the approximate amino acid locations of the characteristic catalytic triad of serine proteases ( $H, D$, and S). $\mathbf{b}$ Human neuropsin preprotein amino acid sequence showing the signal sequence (underlined), the activity-masking peptide (yellow) and endoprotease splice sites (red line). Type 2 neuropsin shows a novel endoprotease site (red arrow) in the type 2-specific amino acid sequence (highlighted in grey)

well as PC12 cells [33]. Its secretion is stimulated by high $\mathrm{K}^{+}$and has been shown to be calcium dependent [33]. The non-active proneuropsin is stored in the extracellular matrix (ECM), probably mostly in the synaptic cleft [34]. Activation of extracellular proneuropsin is facilitated by removal of the activity-masking peptide (QEDK) [35]. The responsible activating endoprotease has not yet been identified, but activation of proneuropsin follows various stimuli in mice, such as kindling epileptogenesis, long-term potentiation (LTP) and application of drugs that can depolarize synaptic activity [34]. Consequently, no or only a little protease activity has been detected in unstimulated brain tissues [2]. Wether neuronal stimuli also initiate secretion of the inactive preproneuropsin is, however, not yet clear. Interestingly, an extra endoprotease site has been identified in the type 2-specific 45-amino acid region (Fig. 3b) causing an intermediate protein form during the activation process [20]. Since protease activation of neuropsin only occurs after cleavage of the activity-masking peptide, this intermediate form shows very low amidolytic activity, like other proneuropsin forms [20].

\section{The neurobiology of neuropsin}

Acting at the synapse, neuropsin has through its proteolytic activity been implicated with several molecular mechanisms underlying synaptic plasticity and associated cognitive and behavioral traits. The coding region of 
$K L K 8$ is relatively conserved between species [36], indicating that the molecular function of neuropsin has been evolutionary maintained. However, dynamic changes in $K L K 8$ TSS usage during primate evolution implies functional divergence of neuropsin in the CNS and speciesspecific roles of neuropsin in neurobiology. Upon its discovery, it was originally speculated that the humanspecific type $2 K L K 8$ may be important for adult brain plasticity, whereas $K L K 8$ in general may be necessary for the development of the human nervous system [26]. However, the detection of type $2 K L K 8$ in the embryo brain [20] complicates such interpretation.

In mice, neuropsin has a regulatory effect on Schaffercollateral LTP [37], which is important for the acquisition of hippocampus-associated memory. LTP describes a persistent strengthening of synapses based on recent patterns of activity and consists of two phases-the temporary early LTP (E-LTP) and the long-lasting late LTP (L-LTP). Whereas L-LTP requires protein kinase A (PKA) activation leading to altered gene expression and novel protein biosynthesis [38], E-LTP comprises an interaction between the ECM and synaptic membranes and thus mechanically modulates synaptic plasticity. It requires $\mathrm{N}$-methyl-D-aspartate (NMDA) receptor and calcium/ calmodulin-dependent protein kinase II (CAMKII) activation, which in turn increases the number of $\alpha$-amino3-hydroxy-5-methyl-4-isoxazolepropionic acid (AMPA) receptors at synapses [39]. Post-synaptic NMDA receptor activation following synaptic stimuli in E-LTP has been shown to induce a rapid activation of the precursor form of neuropsin [4].

Active neuropsin cleaves the extracellular domain of L1CAM and produces a neuropsin-specific $180-\mathrm{kDa}$ fragment $[4,40]$ (depicted in Fig. 4).

The function of this neuropsin-specific L1CAM fragment has not been investigated yet, but the role of cell adhesion molecules in synaptic plasticity [42-45] suggests that neuropsin-specific L1CAM cleavage decreases synaptic adhesion and subsequently increases the flexibility of synaptic structures. Neuropsin is involved in the synaptogenesis of L1CAM expressing orphan and small synaptic boutons in pre-synaptic membranes of the Schaffer-collateral pathway in the hippocampal CA1 substructure [40]. In accordance, Klk8-deficient mice are characterized by abnormalities of synapses and neurons in the CA1 subfield of the hippocampus [46] and show significantly impaired E-LTP and memory acquisition, but not memory retention [47]. Similarly, in vivo inhibition of neuropsin in wild-type (WT) mice leads to impaired E-LTP [47], and delivery of recombinant neuropsin to the hippocampus, conversely, to increased synaptic transmission and AMPA phosphorylation at an E-LTP activating site [47] (depicted in Fig. 4).
Collectively, this implies that neuropsin-dependent L1CAM processing might modulate activity-dependent structural changes involved in E-LTP. It has been suggested that neuropsin, based on its potential of extracellular modulation, might be important for the acquisition of memory depending on input strength [48]. A study in Klk8-deficient mice has demonstrated that neuropsin facilitates the association of two synapses in the apical and basal dendrites of CA1 pyramidal neurons, enabling a weak stimulus resulting in synaptic persistency, when being associated with a separate strongly stimulated pathway [48]. This phenomenon is referred to as synaptic tagging and facilitates the transformation of E-LTP to L-LTP without inducing novel protein biosynthesis.

Another pathway through which neuropsin may contribute to the modulation of synaptic plasticity involves NRG1, its receptor ErbB4 and GABAergic transmission in the CA1-3 subfields of the hippocampus [3] (depicted in Fig. 4). Activated by neuronal activity, neuropsin cleaves and releases NRG1 from its position in the ECM, which subsequently binds to its receptor ErbB4, leading to its phosphorylation [3]. ErbB4 is expressed in parvalbumin-positive GABAergic interneurons within the CA1 region and its phosphorylation is critical for GABAergic transmission [3]. Impairment of GABAergic transmission in Klk8-deficient mice leads to excessive post-synaptic excitation, which prevents the induction of NMDA receptor-dependent LTP [49]. Apart from that, the impairment of parvalbumin-expressing GABAergic interneuron activity contributes to cognitive disturbance which follows seizures as well as asynchronous network activity [50]. Parvalbumin-positive GABAergic interneurons coordinate the excitation/inhibition balance in the hippocampus by generating gamma oscillations $[51,52]$ and it has been found that neuropsin is involved in the generation of slow gamma oscillations via the NRG1ErbB4 pathway [50]. Seizure activity induces neuropsin expression in the mouse brain [53] and has been shown to activate ErbB4-expressing parvalbumin-positive interneurons via neuropsin-NRG1 signaling [50]. Consistently hippocampal gamma oscillations are impaired in neuropsin-KO mice [50].

Linking the function of neuropsin to behavioral regulation, mice exposed to acute or chronic stress show increased $K l k 8$ mRNA expression in hippocampal tissue accompanied by depressive-like behavior [15]. This upregulation of $K l k 8$ after stress exposure was shown to be dependent on the stress hormone corticosterone in primary cultured hippocampal neurons as well as in vivo $[14,15]$. Corticosterone exposure causes impairment in spatial memory, neurogenesis, dendritic morphology and demyelination in WT mice, while mice lacking $K l k 8$ (knockout mice as well as knockdown of $K l k 8$ by viral 


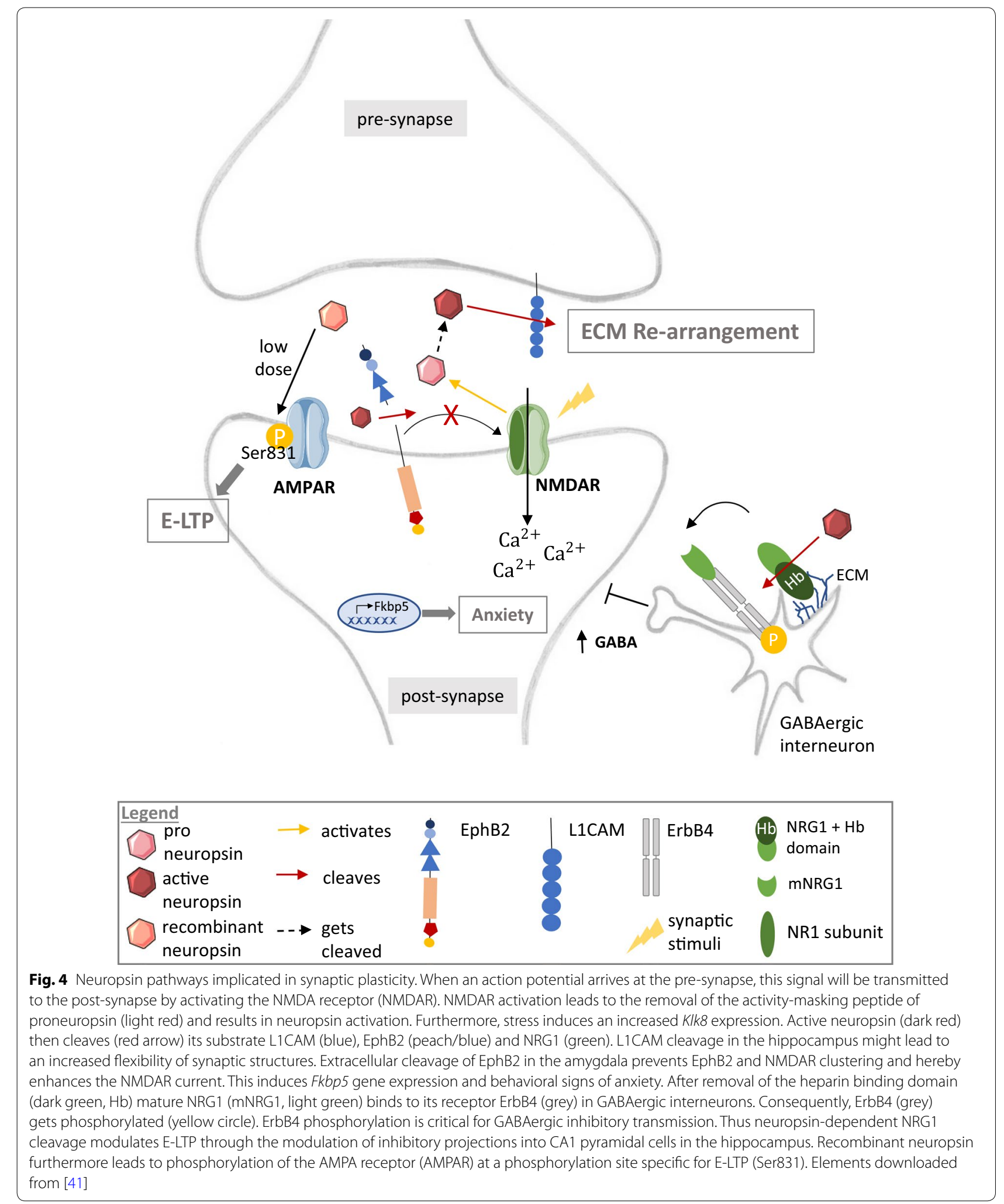


vectors) appear protected against these effects as well as the development of depressive-like behavior [15]. In line with this, overexpression of $K l k 8$ by viral vectors led to an increased impairment in spatial memory and depressivelike behavior. Neuropsin has further been demonstrated to play a critical role in stress-related plasticity in the amygdala, where stress-induced neuropsin-dependent EphB2 cleavage leads to the dissociation of EphB2 from the NR1-subunit of the NMDAR [5]. This changes the dynamics of the EphB2-NMDA-receptor interaction in mice, enhances NMDAR current and induces anxietylike behavior [5]. Klk8-deficient mice, however, are protected against stress-induced EphB2 cleavage and the associated behavioral changes [5].

\section{Klk8 in mental disorders}

Despite its documented role in synaptic signaling and importance in neurobiology and behavior, no clinical data directly link neuropsin to any brain disorder, including mental illnesses. However, there are genetic and epigenetic findings that support its implication in human mental health. This includes clinical data from individuals carrying structural genetic variants encompassing $K L K 8$, among which $>60 \%$ present with intellectual disability [54]. Other associated mental health phenotypes include specific learning disabilities, seizures and autism [54]. However, KLK8 is one of 15 kallikrein subfamily members located in tandem in a gene cluster on chromosome 19 , so phenotypes cannot be specifically attributed to changes in $K L K 8$ copy number. Although a rare $(<0.001 \%$ [55]) missense Val286Ile mutation has been identified in a schizophrenia (SZ) case [56] (Table 1), no other disruptive rare $K L K 8$ variants have been reported in any of the major exome or copy number variation studies performed on SZ or autism spectrum disorder (ASD) cases [57]. Thus, supporting the finding that $K L K 8$ is tolerant to loss of function (LOF) variants $[54,55]$.

A candidate study assessing genetic association between $K L K 8$ polymorphisms and $\mathrm{BD}$ and SZ, identified an association between BD and three SNPs, as well as a two-marker haplotype (Table 1) [56]. Interestingly, healthy individuals carrying the $\mathrm{BD}$ risk allele of $K L K 8$, rs1612902, showed a lower score in attention/concentration and verbal IQ [56]. However, in terms of common variation, no genome-wide significant association could be found between variants in the KLK 8 locus and mental disorders in the currently largest major depressive disorder (MDD), SZ, bipolar disorder (BD), ASD or attention deficit hyperactivity disorder (ADHD) genome-wide association studies (GWASs) [58-62] (Fig. 5 and Additional file 1: Figure S2).

Assessment of $K L K 8$ mRNA levels in peripheral blood from 186 patients diagnosed with major recurrent depression (MRD) compared to 105 healthy subjects, revealed significantly higher $K L K 8$ expression in patients [63]. Furthermore, it was shown that $K L K 8$ mRNA is significantly more abundant in blood samples from patients affected by MRD, compared to patients suffering from first episode depression. The observed increase in $K L K 8$ expression was associated with diminished interpersonal abilities in depressive patients [64]. An association between depression symptomatology score in the general population and blood DNA methylation levels in the promoter region of $K L K 8$ was

Table 1 Descriptive overview of human studies, in which $K L K 8$ was associated to mental health phenotypes

\begin{tabular}{|c|c|c|c|c|}
\hline Phenotype & Tissue & Parameters assessed & Main finding & Reference \\
\hline Schizophrenia & Peripheral blood & $\begin{array}{l}\text { Singe nucleotide polymorphisms (genotyping } \\
\text { assay) }\end{array}$ & $\begin{array}{l}\text { Rare Val286lle missense mutation in exon } 6 \\
\text { detected in a patient with schizophrenia }\end{array}$ & [56] \\
\hline Bipolar disorder & Peripheral blood & $\begin{array}{l}\text { Singe nucleotide polymorphisms (genotyping } \\
\text { assay) }\end{array}$ & $\begin{array}{l}\text { Significant allelic association between several } \\
\text { SNPs and bipolar disorder: } \\
\text { rs1722550 (P=0.019), rs1701946 ( } P=0.018) \\
\text { rs1612902 }(P=0.002), \text { rs1701946 plus } \\
\text { rs1612902 ( } P=0.0068)\end{array}$ & {$[56]$} \\
\hline \multirow[t]{3}{*}{ Depression } & Peripheral blood & $\begin{array}{l}\text { Expression levels (mRNA) of KLK8 (RT-PCR), } \\
\text { patients diagnosed with major recurrent } \\
\text { depression and first episode depression }\end{array}$ & $\begin{array}{l}\text { Higher } K L K 8 \text { expression in patients with recur- } \\
\text { rent depression compared to first episode } \\
\text { patients }\end{array}$ & {$[64]$} \\
\hline & Peripheral blood & $\begin{array}{l}\text { Expression levels (mRNA) of KLK8 (RT-PCR), } \\
\text { patients diagnosed with major recurrent } \\
\text { depression and healthy subjects }\end{array}$ & $\begin{array}{l}\text { Higher KLK8 expression in patients with depres- } \\
\text { sion compared to controls }\end{array}$ & {$[63]$} \\
\hline & Peripheral blood & $\begin{array}{l}\text { Methylation levels of } K L K 8 \text { ( } 450 \text { K methylation } \\
\text { array), depression symptomatology score in } \\
\text { monozygotic twins }\end{array}$ & $\begin{array}{l}\text { Methylation levels in the promotor region of } \\
K L K 8 \text { is associated with depression symptoma- } \\
\text { tology in general population }\end{array}$ & {$[65]$} \\
\hline Alzheimer's disease & $\begin{array}{l}\text { Hippocampal } \\
\text { and parietal } \\
\text { cortex }\end{array}$ & $\begin{array}{l}\text { Expression (RT-PCR), of KLK8 in Alzheimer's } \\
\text { disease (AD) and control tissue }\end{array}$ & $\begin{array}{l}\text { 11.5-fold increase in KLK8 mRNA levels in AD } \\
\text { hippocampus compared to controls }\end{array}$ & [66] \\
\hline
\end{tabular}



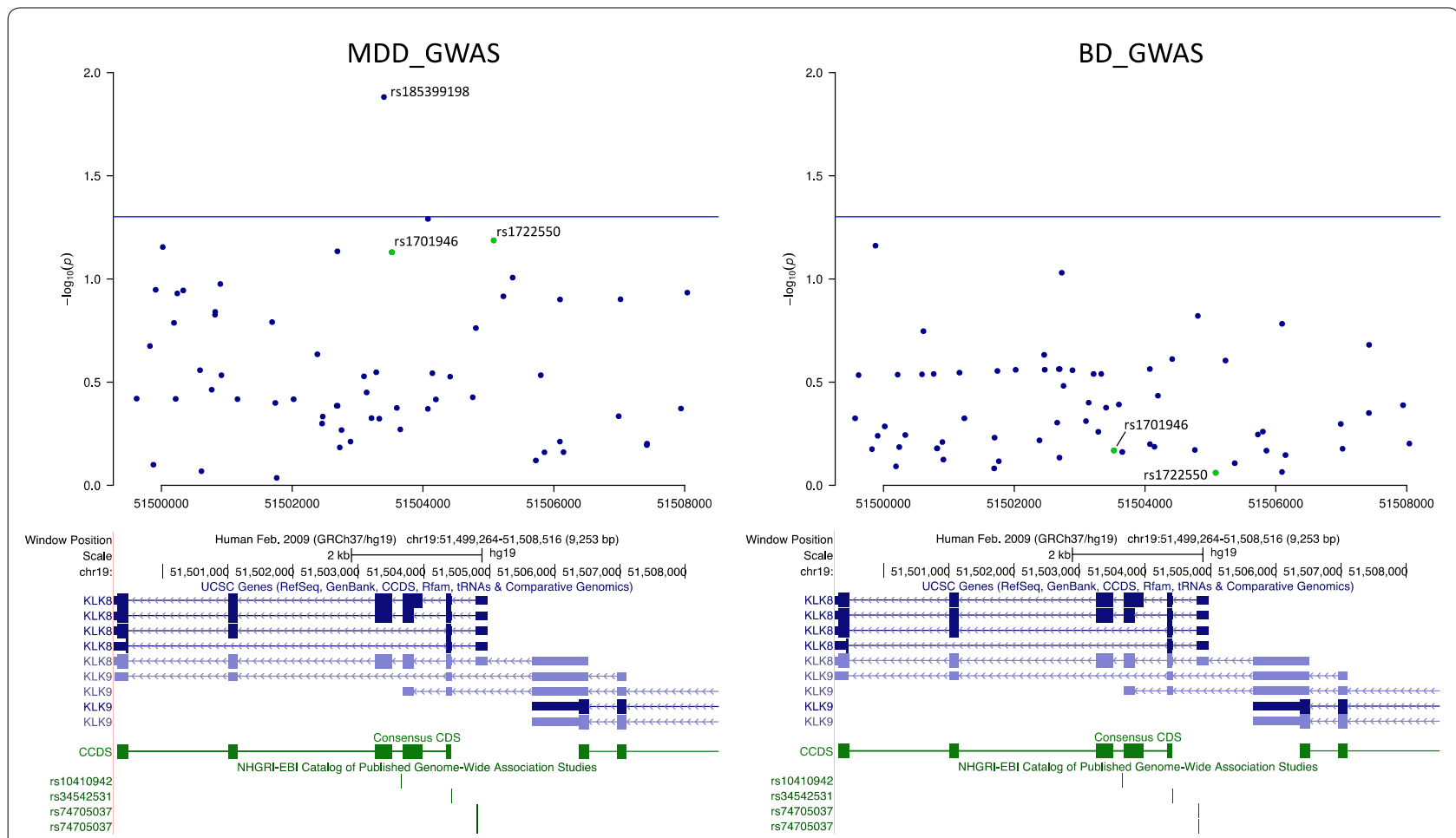

Fig. 5 Single nucleotide polymorphisms (SNPs) associated with mental disorders. Shown are SNPs identified in MDD and BD GWASs [58, 61] as well as two SNPs (rs rs1722550 and rs1701946) associated with bipolar disorder [56] in relation to the UCSC genomic sequences of KLK8 splice variants in blue. Blue line: nominal significant threshold of $p<0.05$. Asterisk indicate SNP located $3^{\prime}$ to KLK8

recently identified in a large cohort of monozygotic Danish twins [65], supporting the implication of $K L K 8$ in depression symptomatology. However, a screening of $K L K 8$ expression data from RNA sequencing expression experiments on post-mortem brain tissues from MDD, BD and SZ patients, did not reveal significant differences between patients and healthy controls in any of the identified studies (Additional file 1: Figures S3 and S4). KLK8 expression levels have, on the other hand, been reported to be 11.5-fold increased in the hippocampus of patients suffering from the neurodegenerative mental disorder Alzheimer's disease (AD) compared to controls [66]. In line with this, RNA sequencing in post-mortem lateral temporal lobe tissue revealed a tendency of higher $K L K 8$ expression in aged AD patients compared to age-matched healthy individuals (GSE104704, Additional file 1: Figure S4).

\section{Limitations}

In this review, we have only assessed available RNA sequencing data, while leaving out data generated from microarray-based platforms. Limited coverage and design of RNA sequencing experiments preclude transcript-specific analyses.

\section{Conclusion}

The proteolytic specificity of neuropsin directed at synaptic proteins implicated in mental disorders, makes it an interesting candidate in molecular psychiatry. Brain-specific splicing of $K L K 8$ mRNA is only reported in humans and its expression and activation characteristics links it to neuronal activity and a neuro-molecular response to stress. Under non-pathological conditions, KLK8 expression in the CNS is, however, weak and appears to be restricted to only a small subset of cells. Through systematic interrogation of available large-scale genetic and post-mortem brain transcriptomic studies, we do not find compelling clinical data that support $K L K 8$ dysregulation in mental illness. However, the endoprotease and molecular machinery responsible for neuropsin activation remains to be characterized and it is possible that post-translational regulation of neuropsin may be the dominating molecular mechanism of activity regulation in the mature brain.

Interestingly, $K L K 8$ expression measured in blood as well as cerebrospinal fluid is a promising early biomarker for $\mathrm{AD}$ as well as mild cognitive impairment due to $\mathrm{AD}$ [67]. Blood KLK8 levels have, furthermore, successfully been established as a biomarker for cancer diagnosis [68]. It is, thus, possible that $K L K 8$ blood parameters levels may 
accordingly serve as diagnostic biomarkers in mental disorders. This is especially interesting when considering that both blood KLK8 mRNA and methylation status have been associated with depression symptomatology $[63,65]$.

\section{Supplementary information}

Supplementary information accompanies this paper at https://doi. org/10.1186/s12576-020-00753-2.

Additional file 1: Table S1. KLK8 expression in human brain single cells. Summarized are KLK8 expression data from several studies [1-4] and databases [5-7]. Figure S1. Amino acid sequence of type 1 (top panel) and type 2 (bottom panel). Figure $\mathbf{S 2}$. Single nucleotide polymorphisms (SNPs) associated with mental disorders. Figure S3. Flowchart describing the dataset search in MEDLINE and GEO. Figure $\mathbf{S 4}$ KLK8 expression in mental disorders.

Additional file 2: Table S2. Output from ModPred. Positions marked in green are positions predicted to feature PTMs.

\section{Abbreviations}

AD: Alzheimer's disease; AMPA: a-Amino-3-hydroxy-5-methyl-4isoxazolepropionic acid; BD: Bipolar disorder; CAMKII: Calcium/calmodulindependent protein kinase II; ECM: Extracellular matrix; E-LTP: Early long-term potentiation; EphB2: Eph receptor B2; GWAS: Genome-wide association study; KLK8: Neuropsin gene; LOF: Loss of function; LTP: Long-term potentiation; L-LTP: Late long-term potentiation; L1CAM: Cell adhesion molecule L1; MDD: Major depressive disorder; MRD: Major recurrent disorder; NMDA: N-MethylD-aspartate; NRG-1: Neuregulin-1; PKA: Protein kinase A; SNP: Single nucleotide polymorphism; SZ: Schizophrenia; WT: Wildtype.

\section{Acknowledgement and funding}

This work was supported by The Lundbeck Foundation Initiative for Integrative Psychiatric Research and Jascha Fonden (case 7662).

\section{Authors' contributions}

Conceptualized the manuscript: LB, AMFC, AS, NHS and PQ. Performed the analyses: $L B, A M F C, A S, N H S$ and $P Q$. Drafted the manuscript: $L B, A S, N H S$, and $P Q$. Provided scientific input and revised the manuscript: $O M, A D B$. All authors read and approved the final manuscript.

\section{Availability of data and materials}

Data are freely available from public repositories with references hereto included in the main text.

\section{Ethics approval and consent to participate}

Not applicable.

\section{Consent for publication}

Not applicable.

\section{Competing interests}

The authors report no biomedical financial interests or potential conflicts of interest.

\section{Author details}

${ }^{1}$ IPSYCH, The Lundbeck Foundation Initiative for Integrative Psychiatric Research, Aarhus, Denmark. ${ }^{2}$ Department of Biomedicine, Aarhus University, Høegh-Guldbergs Gade 10, Aarhus, Denmark. ${ }^{3}$ Center for Genomics and Personalized Medicine, Aarhus University, Aarhus, Denmark. ${ }^{4}$ Psychosis Research Unit, Aarhus University Hospital, Aarhus, Denmark. ${ }^{5}$ Department of Clinical Medicine, Aarhus University, Aarhus, Denmark.

Received: 17 February 2020 Accepted: 6 May 2020

Published online: 15 May 2020

\section{References}

1. Chen ZL et al (1995) Expression and activity-dependent changes of a novel limbic-serine protease gene in the hippocampus. J Neurosci 15(7 Pt 2):5088-5097

2. Shimizu C et al (1998) Characterization of recombinant and brain neuropsin, a plasticity-related serine protease. J Biol Chem 273(18):11189-11196

3. Tamura $\mathrm{H}$ et al (2012) Processing of neuregulin-1 by neuropsin regulates GABAergic neuron to control neural plasticity of the mouse hippocampus. J Neurosci 32(37):12657-12672

4. Matsumoto-Miyai K et al (2003) NMDA-dependent proteolysis of presynaptic adhesion molecule L1 in the hippocampus by neuropsin. J Neurosci 23(21):7727-7736

5. Attwood BK et al (2011) Neuropsin cleaves EphB2 in the amygdala to control anxiety. Nature 473(7347):372-375

6. Hillen AEJ et al (2018) Cell adhesion and matricellular support by astrocytes of the tripartite synapse. Prog Neurobiol 165-167:66-86

7. Xi G et al (2003) The role of thrombin and thrombin receptors in ischemic, hemorrhagic and traumatic brain injury: deleterious or protective? J Neurochem 84(1):3-9

8. Sheehan JJ, Tsirka SE (2005) Fibrin-modifying serine proteases thrombin, tPA, and plasmin in ischemic stroke: a review. Glia 50(4):340-350

9. Tamura $\mathrm{H}$ et al (2013) Does extracellular proteolysis control mammalian cognition? Rev Neurosci 24(4):365-374

10. Mostaid MS et al (2016) Neuregulin-1 and schizophrenia in the genomewide association study era. Neurosci Biobehav Rev 68:387-409

11. Linneberg C et al (2019) L1 cam-mediated developmental processes of the nervous system are differentially regulated by proteolytic processing. Sci Rep 9(1):3716

12. Brainspan - Atlas of the developing human brain. Available from: https:// www.brainspan.org/

13. Konar A, Thakur MK (2015) Neuropsin expression correlates with dendritic marker MAP2c level in different brain regions of aging mice. Mol Neurobiol 51(3):1130-1138

14. Harada A et al (2008) Acute stress increases neuropsin mRNA expression in the mouse hippocampus through the glucocorticoid pathway. Neurosci Lett 436(2):273-277

15. Chang $S$ et al (2016) Neuropsin inactivation has protective effects against depressive-like behaviours and memory impairment induced by chronic stress. PLoS Genet 12(10):e1006356

16. Keyvani Ket al (2018) Higher levels of kallikrein-8 in female brain may increase the risk for Alzheimer's disease. Brain Pathol 28(6):947-964

17. Brainspan-Atlas of the developing human brain

18. Consortium, G (2013) TEx, The Genotype-Tissue Expression (GTEx) project. Nat Genet 45(6):580-585

19. Lu ZX et al (2007) A human-specific mutation leads to the origin of a novel splice form of neuropsin (KLK8), a gene involved in learning and memory. Hum Mutat 28(10):978-984

20. Lu ZX et al (2009) Functional characterization of the human-specific (type II) form of kallikrein 8, a gene involved in learning and memory. Cell Res 19(2):259-267

21. Zhang $Y$ et al (2014) An RNA-sequencing transcriptome and splicing database of glia, neurons, and vascular cells of the cerebral cortex. J Neurosci 34(36):11929-11947

22. La Manno G et al (2016) Molecular Diversity of Midbrain Development in Mouse, Human, and Stem Cells. Cell 167(2):566-580 e19

23. Momota Y et al (1998) Blockade of neuropsin, a serine protease, ameliorates kindling epilepsy. Eur J Neurosci 10(2):760-764

24. Terayama R et al (2004) Differential expression of neuropsin and protease $\mathrm{M} /$ neuropsin in oligodendrocytes after injury to the spinal cord. Glia 48(2):91-101

25. Darmanis S et al (2015) A survey of human brain transcriptome diversity at the single cell level. Proc Natl Acad Sci U S A 112(23):7285-7290

26. Mitsui S et al (1999) A novel form of human neuropsin, a brain-related serine protease, is generated by alternative splicing and is expressed preferentially in human adult brain. Eur J Biochem 260(3):627-634

27. Magklara A et al (2001) The human KLK8 (neuropsin/ovasin) gene: identification of two novel splice variants and its prognostic value in ovarian cancer. Clin Cancer Res 7(4):806-811

28. Sher YP et al (2006) Human kallikrein 8 protease confers a favorable clinical outcome in non-small cell lung cancer by suppressing tumor cell invasiveness. Cancer Res 66(24):11763-11770 
29. Planque $C$ et al (2010) Alternative splicing variant of kallikrein-related peptidase 8 as an independent predictor of unfavorable prognosis in lung cancer. Clin Chem 56(6):987-997

30. Li Y et al (2004) Recent origin of a hominoid-specific splice form of neuropsin, a gene involved in learning and memory. Mol Biol Evol 21(11):2111-2115

31. Shiosaka S, Ishikawa Y (2011) Neuropsin-a possible modulator of synaptic plasticity. J Chem Neuroanat 42(1):24-29

32. ModPred: predictor of post-translational modification sites in proteins. http://www.modpred.org

33. Oka T et al (2002) Role of loop structures of neuropsin in the activity of serine protease and regulated secretion. J Biol Chem 277(17):14724-14730

34. Shiosaka S (2004) Serine proteases regulating synaptic plasticity. Anat Sci Int 79(3):137-144

35. Kishi T et al (2006) Activation and enzymatic characterization of recombinant human kallikrein 8. Biol Chem 387(6):723-731

36. Hecker $\mathrm{N}$ et al (2017) Transition to an aquatic habitat permitted the repeated loss of the pleiotropic KLK8 gene in mammals. Genome Biol Evol 9(11):3179-3188

37. Komai S et al (2000) Neuropsin regulates an early phase of schaffer-collateral long-term potentiation in the murine hippocampus. Eur J Neurosci 12(4):1479-1486

38. Kandel ER (2001) The molecular biology of memory storage: a dialogue between genes and synapses. Science 294(5544):1030-1038

39. Herring BE, Nicoll RA (2016) Long-term potentiation: from CaMKII to AMPA receptor trafficking. Annu Rev Physiol 78:351-365

40. Nakamura Y et al (2006) Role of neuropsin in formation and maturation of Schaffer-collateral L1cam-immunoreactive synaptic boutons. J Cell Sci 119(Pt 7):1341-1349

41. Smart servier medical art. https://smart.servier.com/

42. Benson DL et al (2000) Making memories stick: cell-adhesion molecules in synaptic plasticity. Trends Cell Biol 10(11):473-482

43. Nagappan-Chettiar S et al (2017) Activity-dependent proteolytic cleavage of cell adhesion molecules regulates excitatory synaptic development and function. Neurosci Res 116:60-69

44. Jang S et al (2017) Synaptic adhesion molecules and excitatory synaptic transmission. Curr Opin Neurobiol 45:45-50

45. Sytnyk V et al (2017) Neural cell adhesion molecules of the immunoglobulin superfamily regulate synapse formation, maintenance, and function. Trends Neurosci 40(5):295-308

46. Hirata A et al (2001) Abnormalities of synapses and neurons in the hippocampus of neuropsin-deficient mice. Mol Cell Neurosci 17(3):600-610

47. Tamura $\mathrm{H}$ et al (2006) Neuropsin is essential for early processes of memory acquisition and Schaffer collateral long-term potentiation in adult mouse hippocampus in vivo. J Physiol 570(Pt 3):541-551

48. Ishikawa Y et al (2008) Neuropsin (KLK8)-dependent and -independent synaptic tagging in the schaffer-collateral pathway of mouse hippocampus. J Neurosci 28(4):843-849

49. Moore SD et al (1993) Seizure-like activity disrupts LTP in vitro. Neurosci Lett 163(1):117-119

50. Kawata $\mathrm{M}$ et al (2017) Ablation of neuropsin-neuregulin 1 signaling imbalances ErbB4 inhibitory networks and disrupts hippocampal gamma oscillation. Transl Psychiatry 7(3):e1052
51. Sohal VS et al (2009) Parvalbumin neurons and gamma rhythms enhance cortical circuit performance. Nature 459(7247):698-702

52. Wulff $P$ et al (2009) Hippocampal theta rhythm and its coupling with gamma oscillations require fast inhibition onto parvalbumin-positive interneurons. Proc Natl Acad Sci USA 106(9):3561-3566

53. Okabe A et al (1996) Kindling induces neuropsin mRNA in the mouse brain. Brain Res 728(1):116-120

54. Firth HV et al (2009) DECIPHER: database of chromosomal imbalance and phenotype in humans using ensembl resources. Am J Hum Genet 84(4):524-533

55. Lek M et al (2016) Analysis of protein-coding genetic variation in 60,706 humans. Nature 536(7616):285-291

56. Izumi A et al (2008) Genetic variations of human neuropsin gene and psychiatric disorders: polymorphism screening and possible association with bipolar disorder and cognitive functions. Neuropsychopharmacology 33(13):3237-3245

57. Schizophrenia Exome Sequencing Genebook. http://atgu.mgh.harva rd.edu/ spurcell/genebook/genebook.cgi?user=guest\&cmd=overview

58. Howard DM et al (2019) Genome-wide meta-analysis of depression identifies 102 independent variants and highlights the importance of the prefrontal brain regions. Nat Neurosci 22(3):343-352

59. Demontis D et al (2019) Discovery of the first genome-wide significant risk loci for attention deficit/hyperactivity disorder. Nat Genet 51(1):63-75

60. Grove J et al (2019) Identification of common genetic risk variants for autism spectrum disorder. Nat Genet 51(3):431-444

61. Stahl EA et al (2019) Genome-wide association study identifies 30 loci associated with bipolar disorder. Nat Genet 51(5):793-803

62. Schizophrenia working group of the psychiatric genomics (2014) Consortium, Biological insights from 108 schizophrenia-associated genetic loci. Nature 511(7510):421-427

63. Bobinska K et al (2017) Human neuropsin gene in depression. Psychiatr Danub 29(2):195-200

64. Talarowska M, Bobińska K, Gałecka E, Szemraj J, Gałecki P (2016) Human neuropsin gene and social cognition in depression. Neuropsychiatry 6:396

65. Starnawska A et al (2019) Epigenome-wide association study of depression symptomatology in elderly monozygotic twins. Transl Psychiatry 9(1):214

66. Shimizu-Okabe C et al (2001) Expression of the kallikrein gene family in normal and Alzheimer's disease brain. NeuroReport 12(12):2747-2751

67. Teuber-Hanselmann S et al (2019) CSF and blood Kallikrein-8: a promising early biomarker for Alzheimer's disease. J Neurol Neurosurg Psychiatry 91:40

68. Tailor PD et al (2018) Diagnostic and prognostic biomarker potential of kallikrein family genes in different cancer types. Oncotarget 9(25):17876-17888

\section{Publisher's Note}

Springer Nature remains neutral with regard to jurisdictional claims in published maps and institutional affiliations.

Ready to submit your research? Choose BMC and benefit from

- fast, convenient online submission

- thorough peer review by experienced researchers in your field

- rapid publication on acceptance

- support for research data, including large and complex data types

- gold Open Access which fosters wider collaboration and increased citations

- maximum visibility for your research: over 100M website views per year

At BMC, research is always in progress.

Learn more biomedcentral.com/submissions 